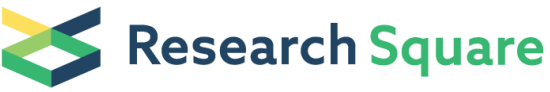 \\ Preprints are preliminary reports that have not undergone peer review. \\ They should not be considered conclusive, used to inform clinical practice, or referenced by the media as validated information.
}

\section{Association between laboratory results on day 8 of onset and the clinical outcome of COVID-19 patients}

\section{Cuilin Shi}

The Affiliated Infectious Diseases Hospital of Soochow University, Suzhou, 215131, P.R.China https://orcid.org/0000-0002-9394-7177

\section{Meilin Shi}

School of Medical Imaging, Xuzhou Medical University, Xuzhou, 221004, P.R.China

Jie Shen

The Affiliated Infectious Diseases Hospital of Soochow University, Suzhou, 215131, P.R.China

Junchi Xu

The Affiliated Infectious Diseases Hospital of Soochow University, Suzhou, 215131, P.R.China

\section{Zhijian Ye}

The Affiliated Infectious Diseases Hospital of Soochow University, Suzhou, 215131, P.R.China

\section{Xiafang Wang}

The Affiliated Infectious Diseases Hospital of Soochow University, Suzhou, 215131, P.R.China

\section{Derui Yan}

Department of Epidemiology and Biostatistics, School of Public Health, Medical College of Soochow University, Suzhou, 215123,

P.R.China.

\section{Lingwu Zeng}

The Affiliated Infectious Diseases Hospital of Soochow University, Suzhou, 215131, P.R.China

\section{Xiujuan Shen}

The Affiliated Infectious Diseases Hospital of Soochow University, Suzhou, 215131, P.R.China

\section{Zaixiang Tang ( $\square$ tangzx@suda.edu.cn)}

Department of Epidemiology and Biostatistics, School of Public Health, Medical College of Soochow University, Suzhou, 215123, P.R.China.

\section{Meiying Wu ( $\sim$ wu_my@126.com )}

The Affiliated Infectious Diseases Hospital of Soochow University, Suzhou, 215131, P.R.China

Keywords: COVID-19, early prognostic biomarkers, clinical outcomes, Lymphocyte count

Posted Date: April 28th, 2020

DOI: https://doi.org/10.21203/rs.3.rs-23964/v1

License: (1) This work is licensed under a Creative Commons Attribution 4.0 International License. Read Full License 


\section{Abstract}

Background: Pneumonia (COVID-19) caused by the novel coronavirus (2019-nCoV) outbreak attracted global attention. However, early prognostic biomarkers on clinical outcomes about COVID-19 patients is not clear by now.

Methods: We collected the clinical data of the 85 COVID-19 patients confirmed in the local hospital. The patients' clinical baseline data, serological data, outcome data were retrospectively analyzed. This study aimed to figure out monitoring values for early prediction of clinical outcome.

Results: Dynamic monitoring of laboratory examination indicators during hospitalization showed that the second week is a critical time window of disease progression. Beginning on the 8th day, severely reduced lymphocytes accompanied by a severe increase in inflammation indicators and abnormally high fibrinogen occurred in serious patients. Plasma fibrinogen was increased $(5.23 \mathrm{vs} 3.87 \mathrm{~g} / \mathrm{L}, \mathrm{P}$ $<0.001)$, and lymphocyte count ( 0.71 vs $1.42109 / \mathrm{L}, \mathrm{P}<0.0001)$ was significantly reduced. On the 8th day of disease course, when the lymphocyte count was lower than $1.23 \times 109 / \mathrm{L}$, the time of negative conversion of viral nucleic acid was prolonged $(P=0.051)$, and imaging showed that the chest CT absorption time was prolonged $(P<0.01)$. When fibrinogen was greater than 4.24 , the time to viral nucleic acid negative conversion was prolonged $(P<0.01)$, and imaging showed that chest $C T$ absorption time was prolonged $(P<0.001)$.

Conclusion: the Lymphocyte count might be related to clinical outcome events. It could act as laboratory indicators of disease severity.

\section{Introduction}

COVID-19 was discovered in Wuhan, China, in December $2019^{1}$. Novel coronavirus is a kind of $\beta$-coronavirus belonging to the genus severe acute respiratory syndrome (SARS)-CoV. Since being declared by the World Health Organization (WHO) as a "public health emergency of international concern" at the end of January 2019, it has spread to nearly 90 countries, with more than 100,000 confirmed cases worldwide, having a great impact on global health, economy, culture, politics, etc. ${ }^{2}$. However, novel coronavirus is extremely infectious, and there have been many studies reporting interpersonal transmission of COVID-19. There have been outbreaks in clusters among those in close contact. The most prominent problems are nosocomial infections and family transmission ${ }^{3-7}$. Several studies have found that its clinical symptoms in the first week of the disease course are similar to those of ordinary upper respiratory tract infections, but the patient's condition may worsen at any time during the second week. Patients who progress to serious disease often do so within 813 days of onset ${ }^{1,4}$. The clinical manifestations, laboratory indicators, and radiological features on the 8th day of disease course may be key to the patient's final clinical outcome ${ }^{4}$. The purpose of this study was to retrospectively analyze the clinical detection indicators of 85 inpatients with COVID-19, dynamically analyze the laboratory detection indicators during hospitalization, compare the correlates of the outcome events of moderate and serious patients, and screen laboratory detection indicators for early prediction of clinical outcomes.

\section{Results}

There were 87 COVID-19 patients in this study. There were 12 groups of clustered onset in families, involving 31 people (35.6\%). Two children of these 31 patients had asymptomatic infections. Considering the special immune status of children under 2 years old, this study excluded the data from these 2 children. According to the clinical classification based on severity defined above, the 85 eligible patients were classified into 68 moderate patients and 17 serious patients.

Of the 85 COVID-19 hospitalized patients, 66 were younger than 60 (77.6\%, range 9-59), 47 were male ( $55.3 \%)$, and 56 (65.9\%) had a Wuhan exposure history. Common symptoms in patients included fever (62 [72.9\%]) and cough (44 [51.8\%]). Less common symptoms were myalgia (5 [5.9\%]) and diarrhea (3 [3.5\%]). Twenty-nine of the 85 patients had been at 12 family gatherings recently. Basic comorbidities included diabetes (4 [4.7\%]), hypertension (7 [8.2\%]), smoking (21 [24.7\%]), and alcohol consumption (3 [3.5\%]). More than $90 \%(90.8 \%, 79 / 87)$ of patients were healthy, showing that people without chronic diseases who contract COVID-19 must be taken seriously. Serious patients were more likely to have persistent fever than moderate patients (16 [94.1\%] vs 46 [67.6\%]). Sixteen of the 17 serious patients had fever symptoms, and $82 \%$ had sustained fever (more than 7 days), indicating that fever persisting for more than one week could be an important clinical symptom giving an early warning of serious disease. One of the serious patients had no fever symptoms throughout the disease course but continued to have hypothermia as a clinical manifestation, which indicates that we cannot ignore the potentially critical patients with normal body temperature. We should stay alert for other possible life-threatening clinical manifestations. Here, $59 \%$ of serious patients had cough symptoms, and a few had symptoms of muscle pain, diarrhea, and vomiting (Table 1). A greater proportion of serious patients had a Wuhan exposure history $(15$ [88.2\%]) vs 41 [60.3\%], $P<0.05)$. There was a greater proportion of male patients among serious patients (12 [70.6\%] vs $36[51.5 \%])$, but this difference was not statistically significant because of the small sample size (Table 1). 
Analysis of baseline clinical laboratory parameters at admission showed that serious patients had higher neutrophil count (3.88 vs $2.67 \times 10^{9}$ cells $/ \mathrm{L}, \mathrm{P}=0.011$ ), higher CRP (14 vs $\left.3.3 \mathrm{~g} / \mathrm{L}, \mathrm{P}=0.003\right)$, higher $\mathrm{PCT}(0.05 \mathrm{vs} 0.03 \mathrm{ng} / \mathrm{mL}, \mathrm{P}=0.016)$, higher plasma fibrinogen (FBG) (4.47 vs $3.91 \mathrm{~g} / \mathrm{L}, \mathrm{P}=0.011$ ), slightly higher serum aspartate aminotransferase (AST) (31 vs $25 \mathrm{U} / \mathrm{L}, \mathrm{P}=0.047$ ), higher myoglobin (MYO) increased (32 vs $21 \mathrm{ng} / \mathrm{mL}, \mathrm{P}=0.014$ ), lower $\mathrm{PaO}_{2} / \mathrm{FiO}_{2}$ (326 vs $420 \mathrm{mmHg}, \mathrm{P}=0.019$ ), and lower lymphocyte count (0.97 vs $\left.1.35 \times 10^{9} / \mathrm{L}, \mathrm{P}<0.0001\right)$ (Table 2).

In this study, two serious patients had severe shock, and two had acute respiratory distress syndrome complications. All patients received antiviral therapy, 26 patients (30.6\%) were treated with low-dose methylprednisolone, and 17 serious patients were treated with hormones. Nasal catheterization was done for all moderate patients, and high-flow nasal cannula oxygen therapy (HFNC) was given to four serious patients, two of whom alternated between HFNC and noninvasive positive-pressure ventilation alternately (Supplemental Table 1)

Based on the dynamic monitoring results of laboratory examinations, we screened out the main clinical features that may occur during the disease course and analyzed the dynamic changes in clinical laboratory parameters (including hematology and biochemical parameters) during hospitalization. The data calibration process for all 85 patients was analyzed. The differences in the WBC, neutrophil count, CRP, and D-dimer between the two groups of patients in the first week of disease were small, but in the second week, the two groups showed significant differences. WBC, neutrophil count, CRP, and D-dimer were higher in serious patients than moderate patients in the second week. Significant lymphocytopenia occurred in the first week of serious disease and became more severe in the second week. From the 8th day of the disease, WBC, neutrophil count, CRP, and D-dimer all increased significantly in serious patients. Significant reductions in lymphocyte count began then. These data show that day 8 of the disease course may be a critical time point for the transition of severity. For patients at this time point, clinical indicators need to be closely and dynamically monitored. In addition, the serum alanine aminotransferase (ALT) and cardiac troponin $\mathrm{T}$ values in serious patients were higher than in moderate patients, suggesting that serious patients are more prone to liver and heart damage (Figure 1 and Supplemental Figure 1).

We excluded patients who had no fever by the 8th day of disease, patients who had passed the 8th day of disease course at the time of admission, and patients with no abnormalities in imaging examination. The data of patients on the 8th (8 \pm 2 ) day were screened out, of whom 59 patients were moderate and 16 patients were serious. The clinical laboratory parameters on the 8th day showed that the neutrophil count was higher in serious patients (3.82 vs $\left.2.5 \times 10^{9} / \mathrm{L}, \mathrm{P}=0.013\right), \mathrm{CRP}$ was higher $(29.2 \mathrm{vs} 2.7 \mathrm{~g} / \mathrm{L}, \mathrm{P}=0.001)$, $\mathrm{PCT}$ was higher (0.05 vs $0.03 \mathrm{ng} / \mathrm{mL}, \mathrm{P}=0.005$ ), plasma FBG was higher (5.23 vs $3.87 \mathrm{~g} / \mathrm{L}, \mathrm{P}<0.001$ ), serum AST was slightly higher (34 vs $27 \mathrm{U} / \mathrm{L}, \mathrm{P}=$ 0.008), MYO was higher (30 vs $21 \mathrm{ng} / \mathrm{mL}, \mathrm{P}=0.017), \mathrm{PaO}_{2} / \mathrm{FiO}_{2}$ was lower (252 vs $\left.420 \mathrm{mmHg}, \mathrm{P}<0.001\right)$, and lymphocyte count was lower $\left(0.71\right.$ vs $\left.1.42 \times 10^{9} / \mathrm{L}, \mathrm{P}<0.0001\right)$ (Table 3$)$.

Correlation analysis between disease severity and the indicators evaluated before discharge showed that normal temperature recovery time was longer in serious patients $(P=0.015)$, the negative conversion period of the viral nucleic acid was longer $(P=0.0088)$, and the $80 \%$ lesion improvement period was longer $(P<0.0001)$ (figure 2$)$.In patients older than 60 , the negative conversion period of viral nucleic acid was longer $(P=0.018)$, and the lesion improvement period on CT imaging was longer $(P=0.032)$, especially in male patients $(P=0.025)$. The lesion improvement period of patients with a Wuhan exposure history was longer, but there was no significant significance because of the small sample size $(P=0.22)$. More than two-thirds of serious patients had a Wuhan exposure history in this study, which indicates that the community isolation and traffic control of Wuhan COVID-19 patients had a positive effect on domestic epidemic control (Figure 3).

The correlations between age, sex, Wuhan exposure history, disease severity, and clinical outcome events were analyzed by univariate analysis. Compared with moderate patients, serious patients had a longer normal temperature recovery time (hazard ratio (HR) $2.05,95 \%$ confidence interval $(\mathrm{Cl}) 1.957-2.147, \mathrm{P}=0.024)$ and longer time until more than $80 \%$ of lung lesions improved (HR 4.448, 95\% $\mathrm{Cl} 4.448-$ $4.48, P=0.047)$. The average time between two negative result tests was also longer ( $\mathrm{HR} 2.046,95 \% \mathrm{Cl} 1.986-2.107, \mathrm{P}=0.015)$. There was no correlation between Wuhan exposure history and clinical outcome events. Patients over 60 years old had a longer time until more than $80 \%$ of lung lesions improved (HR 1.724, 95\% $\mathrm{Cl} 1.573-1.891, \mathrm{P}=0.047)$ and a longer average time between two negative result tests (HR 1.929, 95\% Cl 1.573-1.891, P = 0.023). The time until more than $80 \%$ of lung lesions improved in male patients was longer than in female patients (HR 1.778, 95\% Cl 1.711-1.849, P = 0.02) (Supplemental Figure 2).

We analyzed the clinical laboratory indicators related to disease severity on day 8 and correlated the above-mentioned meaningful clinical indicators with the patient's condition assessment events before discharge. The following laboratory findings on day 8 correlated with a longer time until more than $80 \%$ of lung lesions improved: higher CRP, PCT, plasma FBG, and MYO and lower lymphocyte count. However, there was no significant correlation between WBC and lung lesion improvement time. A prolonged negative result test time by RT-PCR was associated with greater CRP, PCT, and plasma FBG. There was a borderline correlation between lymphocyte count and prolonged negative 
conversion time $(P=0.076)$. There was no correlation between normal temperature recovery time and laboratory test results on day 8 (Supplemental Figure 2).

When lymphocyte count was lower than $1.23 \times 10^{9} / \mathrm{L}$ on day 8 , the negative result test time by RT-PCR was longer $(\mathrm{P}=0.051)$, and chest CT imaging showed that the lesion improvement period was longer $(P=0.00047)$. Patients with a CRP value higher than $29.2 \mathrm{~g} / \mathrm{L}$ had a prolonged negative result test time by RT-PCR $(P=0.017)$ and lesion improvement time $(P=0.0011)$. Patients with $P C T$ above 0.03 ng/mL had a prolonged negative result test time $(P=0.016)$ and lesion improvement time $(P<0.001)$. In patients with platelet value of $242 \times$ $10^{9} / \mathrm{L}$, the negative result test time by RT-PCR $(P=0.0044)$ and the lesion improvement time were prolonged $(P=0.03)$. Patients with plasma FBG greater than $4.24 \mathrm{~g} / \mathrm{L}$ had a prolonged normal temperature recovery time $(P=0.16)$, negative result test time $(P<0.01)$, and lesion improvement time according to CT imaging $(P<0.001)$. WBC had no significant correlation with clinical outcome indicators. These findings suggest that the above-mentioned indicators should be closely and dynamically monitored. CRP and PCT are more sensitive than WBC (Figures $3 a$ and $3 b$ ).

Univariate Cox regression analyses of the laboratory data on 8th day showed that compared with lower lymphocyte count patients, higher lymphocyte count patients had a shorter time until more than $80 \%$ of lung lesions improved $(H R=0.465, P=0.002)$. Further multivariate Cox regression analyses revealed that compared with lower lymphocyte count patients, higher lymphocyte count patients had a shorter time until more than $80 \%$ of lung lesions improved $(\mathrm{HR}=0.482, \mathrm{P}=0.032)$. But no normal temperature recovery time and the negative conversion period of the viral nucleic acid associated with lymphocyte count according to Univariate and multivariate Cox regression analyses $\varangle$ Table $4 \bigotimes$. In conclusion, The lymphocyte count seems to serve as a prognostic factor to predict COVID-19 Patients with delayed Chest CT absorption.

\section{Discussion}

In this study, 17 patients (20\%) were serious, 68 patients (80\%) were moderate, and all patients were safely discharged. This distribution is consistent with the follow-up visit of China CDC, which based on statistics of 72,314 COVID-19 patients ${ }^{8}$. Multi-center research in China by Zhong Nanshan ${ }^{9}$ et al. concluded that the fatality rate of COVID-19 was $1.4 \%$. At the same time, the WHO's estimation was $3.4 \%$, both lower than the SARS-CoV fatality rate of $9.6 \%$ and the MERS-CoV fatality rate of $34.4 \% 10$. However, the fatality rate of serious patients according to research reports varies greatly from region to region $(24.5-48 \%)^{4,11}$. The time of sudden progression from general to serious of most patients is in the second week of disease course, days $8-13$, according to many reports ${ }^{1,4}$. This small-sample study also found that the clinical characteristics on the 8th day were closely correlated with the patient's clinical outcome.

Some $65.9 \%$ of patients in this study had a history of travel to Wuhan or had contact with Wuhan COVID-19 patients (31 patients in 12 families). In one of these families, the grandfather infected five family members during home isolation after taking a train that went through Wuhan. Our research provides further evidence for interpersonal transmission. These findings are consistent with recent reports of the outbreak by family ${ }^{5}$, transmission by asymptomatic individuals ${ }^{12,13}$, and three-phase outbreak patterns ${ }^{14}$. Both COVID-19 patients and asymptomatic patients can transmit 2019-nCoV. Respiratory droplet transmission is the main route of transmission, and the virus can also be transmitted through contact. There is also a risk of aerosol transmission in enclosed spaces. 2019-nCoV has been detected in COVID-19 patients' stool, urine, and blood ${ }^{5}$. Even while the RT-PCR test result is negative, the fecal pathogen nucleic acid test can still be positive ${ }^{5}$. Two young children in this study got infected by their families. Their RT-PCR tests were positive, but they had no symptoms. The whole population is susceptible. Children and infants mostly have mild or moderate disease.

Fever and cough were the main symptoms in our patients with COVID-19, which is consistent with two recent reports ${ }^{5,15}$. COVID-19 patients rarely had gastrointestinal symptoms (such as diarrhea), while approximately $20-25 \%$ of patients infected with MERS-CoV or SARS-CoV had diarrhea ${ }^{16,17}$. The non-fever rate in COVID-19 patients is lower than that in SARS-CoV and MERS-CoV ${ }^{18}$. It is worth noting that in the disease course of serious patients, they could have moderate to low fever, or even have no obvious fever.

Disease severity is an independent predictor of poor prognosis. Our study found that serious patients had prolonged normal temperature recovery time, prolonged negative result test time by RT-PCR, and delayed lung lesion improvement on CT imaging. In this study, patients older than 60 had a prolonged time until more than $80 \%$ of lung lesions improved (HR 1.724, 95\% Cl 1.573-1.891, P = 0.047). The prolonged average time between two negative test results ( HR 1.929, 95\% Cl 1.573-1.891, P $=0.023$ ), and $35.3 \%$ of serious patients were older than 60 , which indicates that advanced age is a risk factor for poor outcomes. Male patients had a longer time until more than $80 \%$ of lung lesions improved (HR 1.778, 95\% Cl 1.711-1.849, $\mathrm{P}=0.02)$, and $70.6 \%$ of serious patients were male, in line with other studies. These data suggest that male sex is a risk factor for serious COIVD-19 infection ${ }^{15}$. 
SRAS-COV2 is pathogenic by binding to angiotensin-converting enzyme 2 (ACE2) receptors on human alveolar type II cells ${ }^{19,20}$. Regarding the difference in sex of serious patients, there may be a certain relationship between higher ACE2 expression in the lungs of Asian men and the higher smoking rate of Chinese men, but this needs to be further verified. The sample size of this study is small, more than $90 \%$ of the patients were healthy, and there were few patients with comorbidities at baseline. Therefore, it is impossible to evaluate the effects of baseline diseases such as diabetes and hypertension on disease severity. However, diabetes, hypertension, and coronary atherosclerotic heart disease can develop into severe disease $e^{4,9}$.

In this study, through dynamic monitoring analysis of laboratory inspection results, we found that the clinical indicators on day 8 were correlated with disease severity and the parameters with significant clinical signs were as follows: higher plasma FBG (5.23 vs $3.87 \mathrm{~g} / \mathrm{L}$, P $<0.001$ ), higher $\mathrm{PaO}_{2} / \mathrm{FiO}_{2}$ (252 vs 420, $\mathrm{P}<0.001$ ), and lower lymphocyte count (0.71 vs 1.42 , P < 0.0001). Significant lymphocytopenia occurred in the first week in serious patients, and it got more severe in the second week. This phenomenon has also been observed in patients with SARS-CoV infection in the past. SARS coronavirus infects the immune system to damage human lung cells circulating immune cells, lymph nodes and spleen. Lymphocytes are the key immune cells in SARS-CoV infection. Lymphopenia is observed in most patients with SARS, and lymphocyte counts typically reach a nadir approximately 2 weeks after onset ${ }^{21}$. The pathological anatomy of the world's first deaths from COVID-19 showed that the main pathological characteristic was diffuse alveolar damage, which is very similar to SARS and MERS coronavirus infection, and T lymphocytes in peripheral blood significantly decreased ${ }^{22}$. Lymphopenia is a common feature in patients with COVID-19 and maybe an independent risk factor related to disease severity and fatality.

WBC, neutrophil count, CRP, and plasma FBG showed relatively small differences in the first week of the disease course in both groups, but there were significant differences in the second week. In serious patients, apart from the significant decrease in lymphocyte count, other indicators, such as WBC, neutrophil count, CRP, and plasma FBG increased significantly from the eighth day of the disease course. Inflammation- and infection-related indicators increased sharply at 2 weeks after onset, and the number of immune cells in the body decreased sharply due to a large number of viruses, which may be because airway-colonizing bacteria multiply after the body's immune function is suppressed. It could also be related to the cytokine storm caused by virus invasion. When COVID-19 patients are coinfected, the sensitivity of CRP is high. Appropriate anti-infective treatment should be delivered as soon as possible. Correlation analysis between laboratory indicators on the 8th day of disease course and clinical outcomes in this study found that patients with lymphocyte count less than $1.23 \times 10^{9} / \mathrm{L}$ and CRP higher than $29.2 \mathrm{~g} / \mathrm{L}$ on day 8 of onset had a prolonged lung lesion improvement time and negative result test time by RT-PCR. Multivariate Cox regression and further analysis indicated that the lymphocyte count as a prognostic factor to predict the delay of chest CT absorption in COVID-19 patients after adjustment for multiple risk factors $(\mathrm{P}<0.032)$.

All serious patients in this study had coagulopathy, which was mainly manifested by increased D-dimer and plasma FBG. Some of the serious patients' conditions suddenly worsened during the disease course. In previous studies of patients with COVID-19, D-dimer was significantly higher in ICU patients than moderate patients ${ }^{1,4}$. Ribelles ${ }^{17}$ found that D-dimer $>500 \mu \mathrm{g} / \mathrm{L}$ had a strong correlation with death in patients with community-acquired pneumonia. The reason was that inflammatory mediators tend to promote coagulation after imbalance of coagulation and fibrinolysis in alveoli, which activates the fibrinolytic system and then cause D-dimer to increase. In this study, D-dimer was also slightly increased in moderate patients, but not statistically significantly. For the early prediction of COVID19 patients, it was more significant that plasma FBG value was significantly higher in serious patients on the 8th day of disease course (5.23 vs 3.87, $\mathrm{P}<0.001)$. This study found that in patients with plasma FBG values greater than $4.24 \mathrm{~g} / \mathrm{L}$ on the 8 th day, the negative RT-PCR test time $(P=0.0028)$, and the time until lung lesions improved were longer $(P=0.000086)$. At present, there are no data on the dynamic evolution of coagulation indices in serious patients in rehabilitation. Analysis with large sample size is needed to determine the correlation between coagulation indices and the severity of COVID-19 patients to facilitate early intervention and correction. Serious type patients in this study were more prone to heart injury than moderate patients, which was consistent with the findings of others ${ }^{1}$. This study also found that patients with MYO greater than $21 \mathrm{ng} / \mathrm{mL}$ had a longer lesion improvement period. The cause of myocardial damage in serious patients with COVID-19 may be related to the more severe lung injury with persistent hypoxemia, which leads to unstable myocardial electrical signals.

There are currently no specific treatment or prevention protocols, such as targeted antiviral drugs or vaccines, for COVID-19. Therefore, China has focused on traditional public health outbreak response models, such as controlling the source of infection, recommending personal protective measures to reduce transmission risk, early diagnosis, isolation, and supportive care for infected patients ${ }^{23}$. As a designated hospital for COVID-19 treatment, our hospital strictly implements the highest level of personal protection, and none of the hospital's medical workers are infected. Despite the controversy over the negative effects of such stringent community isolation measures, China's COVID-19 infection rate fell rapidly from $5.6 \%$ to $0.4 \%$. 
This study has several limitations. First, oropharyngeal swabs were used to diagnose COVID-19 by RT-PCR, and no patient serum was obtained to assess viral load or viral antibodies such as lgM and lgG. The viral load is a potentially useful marker that is related to disease severity. The IgM and IgG test is a good complement to the RT-PCR test. It is of great significance for the diagnosis, treatment monitoring, and outcome of the disease and should be evaluated before discharge. Second, the cytokine storm is a prominent problem in serious patients, but this study did not measure cytokines. Third, the sample size was small, and we failed to do a multi-factor analysis of the key laboratory indicators and clinical outcome events on the 8th day. Fourth, the patients were mainly moderate, and family-cluster onset accounted for more than one-third of the cases, so the data provided may not be completely representative.

\section{Conclusion}

Disease severity is an independent predictor of poor prognosis. Advanced age and male sex are risk factors for COVID-19 severity. The second week of disease course may be a critical period for exacerbation. It is necessary to pay specific attention to lymphocyte count, plasma $\mathrm{FBG}, \mathrm{CRP}$, and $\mathrm{PaO}_{2} / \mathrm{FiO}_{2}$ on day 8 . Early identification of patients with severe disease tendency is important. Pulse oxygen index should be monitored closely, and CT scans should be assessed for these patients to provide necessary supportive treatment in time. On day 8 of the disease course, patients with a lymphocyte count lower than $1.23 \times 10^{9} / \mathrm{L}$ and plasma FBG higher than $4.24 \mathrm{~g} / \mathrm{L}$ may have longer negative result test time and lesion improvement period.

\section{Methods}

\section{Study design and participants}

This hospital is the designated COVID-19 hospital in Suzhou, Jiangsu Province, China. The patients included in this study were all COVID19 patients diagnosed by the Suzhou Center for Disease Control (CDC) laboratory according to the interim guidelines of the WHO. The hospitalization date of these patients was from January 21,2019 , to March 10,2020 . All patients were discharged successfully before March 10. This study followed the hospital's ethics and regulations.

\section{Data collection}

Patient information reviewed and recorded by a team of trained physicians included epidemiological data, medical history, contact history, potential comorbidities, symptoms, signs, laboratory results, chest computed tomography (CT) scans, treatment measures (i.e., antiviral therapy, glucocorticoid therapy, respiratory support, and other treatments), and evaluation data related to clinical outcomes of patients before discharge (clinical classification of the patient's final condition, $80 \%$ CT absorption time, normal temperature recovery time, the negative result test time of oropharyngeal swab, and cough improvement time). The onset date was defined as the date on which symptoms appeared.

\section{Detection of coronavirus in oropharyngeal swabs}

Reverse transcription-real-time polymerase chain reaction (RT-PCR) was done to extract 2019-nCoV RNA from 2019-nCoV-infected patients. An oropharyngeal swab was placed in a collection tube containing $150 \mu \mathrm{L}$ of the virus preservation solution and extracted using a respiratory sample nucleic acid extraction kit (Cat \# 6543588001, Roche) within 2 hours. For specific experimental methods, please refer to (5).

\section{Definitions}

The diagnostic criteria of the clinical classification of "Novel Coronavirus Pneumonia Diagnosis and Treatment Plan (Provisional 7th Edition)" issued by the National Health Commission of China divide COVID-19 into four types: (1) Mild: clinical symptoms are mild, with no pneumonia manifestations on imaging. (2) Moderate: fever, respiratory tract symptoms, etc.; imaging shows pneumonia. (3) Serious: any one of the following: respiratory distress, defined as respiratory rate $\geq 30$ breaths $/ \mathrm{min}$; blood oxygen saturation $\leq 93 \%$ at rest; or arterial blood oxygen pressure $\left(\mathrm{PaO}_{2}\right)$ /oxygen concentration $\left(\mathrm{FiO}_{2}\right) \leq 300 \mathrm{mmHg}$. (4) Critically ill: any one of the following: respiratory failure requiring mechanical ventilation; shock; or combined with other organ failure requiring ICU care. In this study, we defined $\mathrm{mild} /$ moderate patients as moderate and serious/critically ill patients as serious. 


\section{Statistical analysis}

Patients were divided into two groups according to disease severity (mild/moderate, serious/critically ill), and the measurement data are statistically described by $\mathrm{x} \pm \mathrm{s}$ or $\mathrm{M}(\mathrm{Q} 2-\mathrm{Q} 4)$. The count data were compared between groups with the chi-squared test, $t$ test or Wilcoxon rank sum test.

We screened out the patients' data on the 8th $(8 \pm 2)$ day of the disease course. We dichotomized white blood cell count (WBC), lymphocytes, C-reactive protein(CRP), procalcitonin (PCT), platelets, D-dimer, and myoglobin (MYO) around the median. We used a Cox regression model to analyze the relationship between each outcome $₫ 80 \%$ chest Computed Tomography absorption time, normal body temperature recovery time, the negative result test time of oropharyngeal swab, and each biochemical index. The log-rank test and KaplanMeier accumulated survival curves were used to compare the cumulative risk between the two groups. Statistical analysis was performed using the "survival", "rms", and "survminer" packages in R software. $\mathrm{P}<0.05$ indicated a statistically significant difference. Missing values were calculated after multiple filling by mice package.

\section{Declarations}

\section{Acknowledgments}

This work was supported by the National Natural Science Foundation of China (81773541 and 81573253 ), and Suzhou city Science and Technology Plan Project ( SYSD2018193, SS2019010, and SYS2019111). Our research sponsors had no role in the research design, data collection, data analysis, data interpretation, or report writing. The corresponding author had full access to all data in the study and takes responsibility for the final decision to submit it for publication.

\section{Conflict of interests}

All authors: No reported conflicts.

\section{Contributions}

(I) Conception and design: Meiying Wu, Zaixiang Tang; (II) Administrative support: Meiying Wu, Zaixiang Tang; (III) Provision of study materials or patients: Cuilin Shi, Meilin Shi, Jie Shen, Junchi Xu, Zhijian Ye, Xiafang Wang, Derui Yan, Peijun Tang, Lingwu Zeng, Xiujuan Shen; (IV) Collection and assembly of data: Cuilin Shi, Meilin Shi, Jie Shen; (V) Data analysis and interpretation: Cuilin Shi, Derui Yan, Junchi Xu; (VI) Manuscript writing: All authors; (VII) Final approval of manuscript: All authors.

\section{Ethics}

Ethical approva/ was achieved from the Affiliated Infectious Diseases Hospital of Soochow University Hospital ethical committee. Patients or surrogates signed informed consent.

\section{References}

1 C, H. et al. Clinical features of patients infected with 2019 novel coronavirus in Wuhan, China. Lancet (London, England) 395, 497506, doi:10.1016/s0140-6736(20)30183-5 (2020).

2 C, S. et al. World Health Organization declares Global Emergency: A review of the 2019 Novel Coronavirus (COVID-19). International journal of surgery (London, England), doi:10.1016/j.ijsu.2020.02.034 (2020).

3 Parry, J. China coronavirus: cases surge as official admits human to human transmission. BMJ (Clinical research ed.) 368, m236, doi:10.1136/bmj.m236 (2020).

4 D, W. et al. Clinical Characteristics of 138 Hospitalized Patients With 2019 Novel Coronavirus-Infected Pneumonia in Wuhan, China. JAMA, doi:10.1001/jama.2020.1585 (2020).

5 Chan, J. F. et al. A familial cluster of pneumonia associated with the 2019 novel coronavirus indicating person-to-person transmission: a study of a family cluster. Lancet, doi:10.1016/s0140-6736(20)30154-9 (2020).

6 Zhu, N. et al. A Novel Coronavirus from Patients with Pneumonia in China, 2019. N Engl J Med, doi:10.1056/NEJMoa2001017 (2020). 
7 Phan, L. T. et al. Importation and Human-to-Human Transmission of a Novel Coronavirus in Vietnam. N Engl J Med, doi:10.1056/NEJMc2001272 (2020).

8 Wu Z, M. J. Characteristics of and Important Lessons From the Coronavirus Disease 2019 (COVID-19) Outbreak in China: Summary of a Report of 72314 Cases From the Chinese Center for Disease Control and Prevention. JAMA, doi:10.1001/jama.2020.2648.

9 WJ, G. et al. Clinical Characteristics of Coronavirus Disease 2019 in China. The New England journal of medicine, doi:10.1056/NEJMoa2002032 (2020).

10 NC, P. et al. The SARS, MERS and novel coronavirus (COVID-19) epidemics, the newest and biggest global health threats: what lessons have we learned? International journal of epidemiology, doi:10.1093/ije/dyaa033 (2020).

11 C, C. et al. The novel zoonotic COVID-19 pandemic: An expected global health concern. Journal of infection in developing countries 14, 254-264, doi:10.3855/jidc.12671 (2020).

12 C, R. et al. Transmission of 2019-nCoV Infection from an Asymptomatic Contact in Germany. The New England journal of medicine 382, 970-971, doi:10.1056/NEJMc2001468 (2020).

13 C, D. R. \& PN, M. 2019 Novel Coronavirus-Important Information for Clinicians. JAMA, doi:10.1001/jama.2020.1490 (2020).

14 Q, L. et al. Early Transmission Dynamics in Wuhan, China, of Novel Coronavirus-Infected Pneumonia. The New England journal of medicine, doi:10.1056/NEJMoa2001316 (2020).

15 N, C. et al. Epidemiological and clinical characteristics of 99 cases of 2019 novel coronavirus pneumonia in Wuhan, China: a descriptive study. Lancet (London, England) 395, 507-513, doi:10.1016/s0140-6736(20)30211-7 (2020).

16 WK, L. et al. Enteric involvement of severe acute respiratory syndrome-associated coronavirus infection. Gastroenterology 125, 1011-1017, doi:10.1016/s0016-5085(03)01215-0 (2003).

17 Y, W., Y, W., Y, C. \& Q, Q. Unique epidemiological and clinical features of the emerging 2019 novel coronavirus pneumonia (COVID-19) implicate special control measures. Journal of medical virology, doi:10.1002/jmv.25748 (2020).

18 ZA, M., S, P., MD, V. K. \& A, Z. Middle East respiratory syndrome. Lancet (London, England), doi:10.1016/s0140-6736(19)33221-0 (2020).

$19 \mathrm{M}, \mathrm{H}$. et al. SARS-CoV-2 Cell Entry Depends on ACE2 and TMPRSS2 and Is Blocked by a Clinically Proven Protease Inhibitor. Cell, doi:10.1016/j.cell.2020.02.052 (2020).

20 R, Y. et al. Structural basis for the recognition of the SARS-CoV-2 by full-length human ACE2. Science (New York, N.Y.), doi:10.1126/science.abb2762 (2020).

21 Guo, Y., Korteweg, C., McNutt, M. A. \& Gu, J. Pathogenetic mechanisms of severe acute respiratory syndrome. Virus research 133, 412, doi:10.1016/j.virusres.2007.01.022 (2008).

22 Z, X. et al. Pathological findings of COVID-19 associated with acute respiratory distress syndrome. The Lancet. Respiratory medicine, doi:10.1016/s2213-2600(20)30076-x (2020).

23 A, W.-S. \& DO, F. Isolation, quarantine, social distancing and community containment: pivotal role for old-style public health measures in the novel coronavirus (2019-nCoV) outbreak. Journal of travel medicine, doi:10.1093/jtm/taaa020 (2020).

\section{Tables}

Table 1. Baseline Characteristics of COVID-19 patients 


\begin{tabular}{|c|c|c|c|c|c|}
\hline \multicolumn{6}{|l|}{$\begin{array}{l}\mathrm{N} \\
\text { Age }\end{array}$} \\
\hline $9-59$ & 55 & 11 & 66 & 1.224 & 0.269 \\
\hline $60-70$ & 13 & 6 & 19 & & \\
\hline \multicolumn{6}{|l|}{$\operatorname{sex}$} \\
\hline male & 35 & 12 & 47 & 2.011 & 0.156 \\
\hline female & 33 & 5 & 38 & & \\
\hline \multicolumn{6}{|l|}{ exposure } \\
\hline exposure to Wuhan & 41 & 15 & 56 & 4.724 & 0.0298 \\
\hline None exposure to Wuhan & 27 & 2 & 29 & & \\
\hline \multicolumn{6}{|l|}{ chronic medical illness } \\
\hline hypertension & 5 & 2 & 7 & 0.01 & 0.921 \\
\hline None hypertension & 63 & 15 & 78 & & \\
\hline $2-\mathrm{DM}$ & 2 & 2 & 4 & & 0.177 \\
\hline None 2-DM & 66 & 15 & 81 & & \\
\hline smoking & 14 & 7 & 21 & 2.091 & 0.148 \\
\hline None smoking & 54 & 10 & 64 & & \\
\hline drinking & 1 & 2 & 3 & & 0.101 \\
\hline None drinking & 67 & 15 & 82 & & \\
\hline \multicolumn{6}{|l|}{ symptoms } \\
\hline fever & 46 & 16 & 62 & 3.58 & 0.058 \\
\hline None fever & 22 & 1 & 23 & & \\
\hline cough & 34 & 10 & 44 & 0.424 & 0.515 \\
\hline None cough & 34 & 7 & 41 & & \\
\hline myalagia & 3 & 2 & 5 & & 0.26 \\
\hline None myalagia & 65 & 15 & 81 & & \\
\hline diarrhea & 3 & 0 & 3 & & 1 \\
\hline None diarrhea & 65 & 17 & 82 & & \\
\hline
\end{tabular}

Table 2. Laboratory data of COVID-19 patients on Admission to Hospital

\begin{tabular}{|c|c|c|c|c|c|}
\hline & $\begin{array}{l}\text { mild/moderate } \\
\text { patients }\end{array}$ & $\begin{array}{l}\text { serious / critically ill } \\
\text { patients }\end{array}$ & All patients & Statistical & $\mathrm{p}$ \\
\hline $\bar{N}$ & 68 & 17 & 85 & & \\
\hline White blood cell count(WBC), 10^9/L & $4.78(3.38-6.12)$ & $5.42(3.98-6.91)$ & $4.9(3.49-6.2)$ & -1.29 & 0.197 \\
\hline Neutrophil count(N), 10^9/L & $2.67(1.9-3.69)$ & $3.88(2.75-5.9)$ & $2.76(2.01-3.88)$ & -2.55 & 0.011 \\
\hline Lymphocyte count(L), 10^9/L & $1.35(1.04-1.73)$ & $0.97(0.66-1)$ & $1.18(0.97-1.64)$ & -3.99 & $<0.01$ \\
\hline Platelet count(PLT), 10^9/L & $194.5(115.75-241.5)$ & $138(138-138)$ & 184.54(88.91) & -0.63 & 0.529 \\
\hline C-reactive protein(CPR), $\mathrm{g} / \mathrm{L}$ & $3.3(0.5-16.5)$ & $14(7.2-29.2)$ & $6.7(0.8-17.6)$ & -3.02 & 0.003 \\
\hline Procalcitonin(PCT), ng/mL & $0.03(0.02-0.05)$ & $0.05(0.03-0.07)$ & $0.04(0.02-0.05)$ & -2.41 & 0.016 \\
\hline $\mathrm{D}$-dimer, $\mu \mathrm{g} / \mathrm{L}$ & $180(137.5-262.5)$ & $239(165-340)$ & $190(140-285)$ & -1.65 & 0.098 \\
\hline Plasma fibrinogen (FBG), $\mathrm{g} / \mathrm{L}$ & $3.91(2.99-4.5)$ & $4.47(4.11-5.07)$ & $4.11(3.21-4.58)$ & -2.54 & 0.011 \\
\hline Total billirubin(TB), $\mu \mathrm{mol} / \mathrm{L}$ & $9.6(6.85-14.8)$ & $14.65(10.83-18.48)$ & $9.6(7-15.2)$ & -0.45 & 0.65 \\
\hline $\begin{array}{l}\text { Serum glutamic pyruvic transaminase(ALT), } \\
\text { U/L }\end{array}$ & $29(24.5-38)$ & $31(24-48)$ & $29(24-42.5)$ & -0.66 & 0.507 \\
\hline $\begin{array}{lcc}\text { Serum } & \text { glutamic } & \text { oxaloacetic } \\
\text { transaminase(AST), U/L } & \end{array}$ & $25(21-32.5)$ & $31(25-46)$ & $\begin{array}{l}27(21.75- \\
33.25)\end{array}$ & -1.98 & 0.047 \\
\hline Cardiac troponin T $\square c T n T \square p g / m L$ & $3(3-6)$ & $4(3-5)$ & $4(3-6)$ & -0.30 & 0.764 \\
\hline CKMB & $0.6(0.41-1.04)$ & $0.82(0.31-1.16)$ & $0.61(0.41-1.13)$ & -0.12 & 0.908 \\
\hline Myoglobin(MYO), ng/mL & $21(21-29.88)$ & $32.23(24.53-47.29)$ & 23.4(21-33.26) & -2.46 & 0.014 \\
\hline Serum creatinine concentration $(\mathrm{Cr}), \mu \mathrm{mol} / \mathrm{L}$ & $62.88(19.36)$ & $70.51(13.56-0)$ & $64.48(18.49)$ & -1.44 & 0.154 \\
\hline $\mathrm{PaO} 2 / \mathrm{FiO} 2$ & $420(354.5-487.5)$ & $326(270-440)$ & $418.41(108.79)$ & -2.34 & 0.019 \\
\hline
\end{tabular}

Table3. Laboratory data of COVID-19 patients on the 8th onset 


\begin{tabular}{|c|c|c|c|c|c|}
\hline & $\begin{array}{l}\text { mild/moderate } \\
\text { patients }\end{array}$ & critically ill & All patients & Stastical & $\mathrm{p}$ \\
\hline$\overline{\mathrm{N}}$ & 59 & 16 & 75 & & \\
\hline White blood cell count(WBC), 10^9/L & $4.61(3.6-6.16)$ & $4.64(4.12-8.35)$ & $4.62(3.8-6.22)$ & -0.92 & 0.361 \\
\hline Neutrophil count(N), 10^9/L & $2.5(1.89-3.48)$ & $3.82(2.95-7.26)$ & $2.95(2.01-3.87)$ & -2.50 & 0.013 \\
\hline Lymphocyte count(L), $10^{\wedge} 9 / \mathrm{L}$ & $1.42(1.09-1.8)$ & $0.71(0.57-0.89)$ & $1.23(0.88-1.65)$ & -4.92 & 0 \\
\hline Platelet count(PLT), 10^9/L & $227.5(161.5-271)$ & $320(284-331)$ & $242(192-290)$ & -3.08 & 0.002 \\
\hline C-reactive protein(CPR), $\mathrm{g} / \mathrm{L}$ & $2.7(0.5-18.2)$ & $29.2(15.85-41)$ & $\begin{array}{l}7.25(0.53- \\
24.55)\end{array}$ & -3.26 & 0.001 \\
\hline Procalcitonin(PCT), ng/mL & $0.03(0.02-0.04)$ & $0.05(0.03-0.08)$ & $0.03(0.02-0.05)$ & -2.83 & 0.005 \\
\hline D-dimer, $\mu \mathrm{g} / \mathrm{L}$ & $200(140-280)$ & $250(205-385)$ & $210(140-280)$ & -1.70 & 0.09 \\
\hline Plasma fibrinogen (FBG), g/L & $3.87(3.1-4.8)$ & $5.23(4.52-6.4)$ & $4.24(3.2-5.04)$ & -3.63 & 0 \\
\hline Total billirubin(TB), $\mu \mathrm{mol} / \mathrm{L}$ & $10.9(7.3-13)$ & $10(8.25-12.75)$ & $10.65(7.63-13)$ & -0.03 & 0.982 \\
\hline $\begin{array}{l}\text { serum glutamic pyruvic transaminase(ALT), } \\
\mathrm{U} / \mathrm{L}\end{array}$ & $31(25-39.5)$ & $41.5(27.75-62.5)$ & $32(25-44)$ & -1.35 & 0.178 \\
\hline $\begin{array}{lcc}\text { serum } & \text { glutamic } & \text { oxaloacetic } \\
\text { transaminase(AST), U/L } & \end{array}$ & $27(20-34)$ & $34(26.75-51.5)$ & $28(22-37)$ & -2.65 & 0.008 \\
\hline Cardiac troponin $\mathrm{T} \square \mathrm{cTnT} \square \mathrm{pg} / \mathrm{mL}$ & $3(3-5)$ & $4(3-5)$ & $3(3-5)$ & -0.77 & 0.443 \\
\hline CKMB & $0.46(0.3-0.6)$ & $0.5(0.3-1.04)$ & $0.48(0.3-0.71)$ & -0.56 & 0.579 \\
\hline Myoglobin(MYO), ng/mL & $21(21-29.79)$ & $30(22.64-48.45)$ & $21(21-33.09)$ & -2.40 & 0.017 \\
\hline Serum creatinine concentration $(\mathrm{Cr}), \mu \mathrm{mol} / \mathrm{L}$ & $58.05(45.93-77.45)$ & $70.6(56.53-75.58)$ & $\begin{array}{l}60.55(46.7- \\
77.15)\end{array}$ & -1.20 & 0.235 \\
\hline $\mathrm{PaO} 2 / \mathrm{FiO} 2$ & $420(339-510)$ & $252(210-304.25)$ & $397.71(122.87)$ & -4.91 & $<0.01$ \\
\hline
\end{tabular}

Table 4. Univariate and multivariate Cox regression analyses of lymphocyte count on 8th onset

\begin{tabular}{lccccc}
\hline \multirow{2}{*}{ Index } & $\begin{array}{c}80 \% \text { chest CT absorption } \\
\text { time }\end{array}$ & Normal body temperature recovery & \multicolumn{2}{c}{ The negative result test time of oropharyngeal } \\
& HR $(95 \% \mathrm{CI})$ & $\mathrm{P}$ & HR $(95 \% \mathrm{CI})$ & $\mathrm{P}$ & $\mathrm{HR}(95 \% \mathrm{CI})$ \\
\hline $\begin{array}{l}\text { Model } \\
1\end{array}$ & $0.465(0.289-0.751)$ & 0.002 & $0.552(0.272-1.122)$ & 0.101 & $0.652(0.406-1.046)$ \\
Model & $0.482(0.247-0.938)$ & 0.032 & $0.433(0.145-1.295)$ & 0.134 & $0.506(0.256-1.001)$ \\
2
\end{tabular}

Model 1, Univariate Cox regression analyses; Model 2, Multivariate Cox regression analyses $₫$ the adjusted factors are age, gender, Wuhan exposure history, white blood cell count, c-reactive protein, procalcitonin, platelet count, plasma fibrinogen, myoglobin; Model 3, Multivariate Cox regression analyses $\square$ the adjusted factors are age, gender, Wuhan exposure history, disease severity, white blood cell count, c-reactive protein, procalcitonin, platelet count, plasma fibrinogen, myoglobin. lymphocyte count threshold was $1.23 \times 10^{9} / \mathrm{L}$.

\section{Figures}



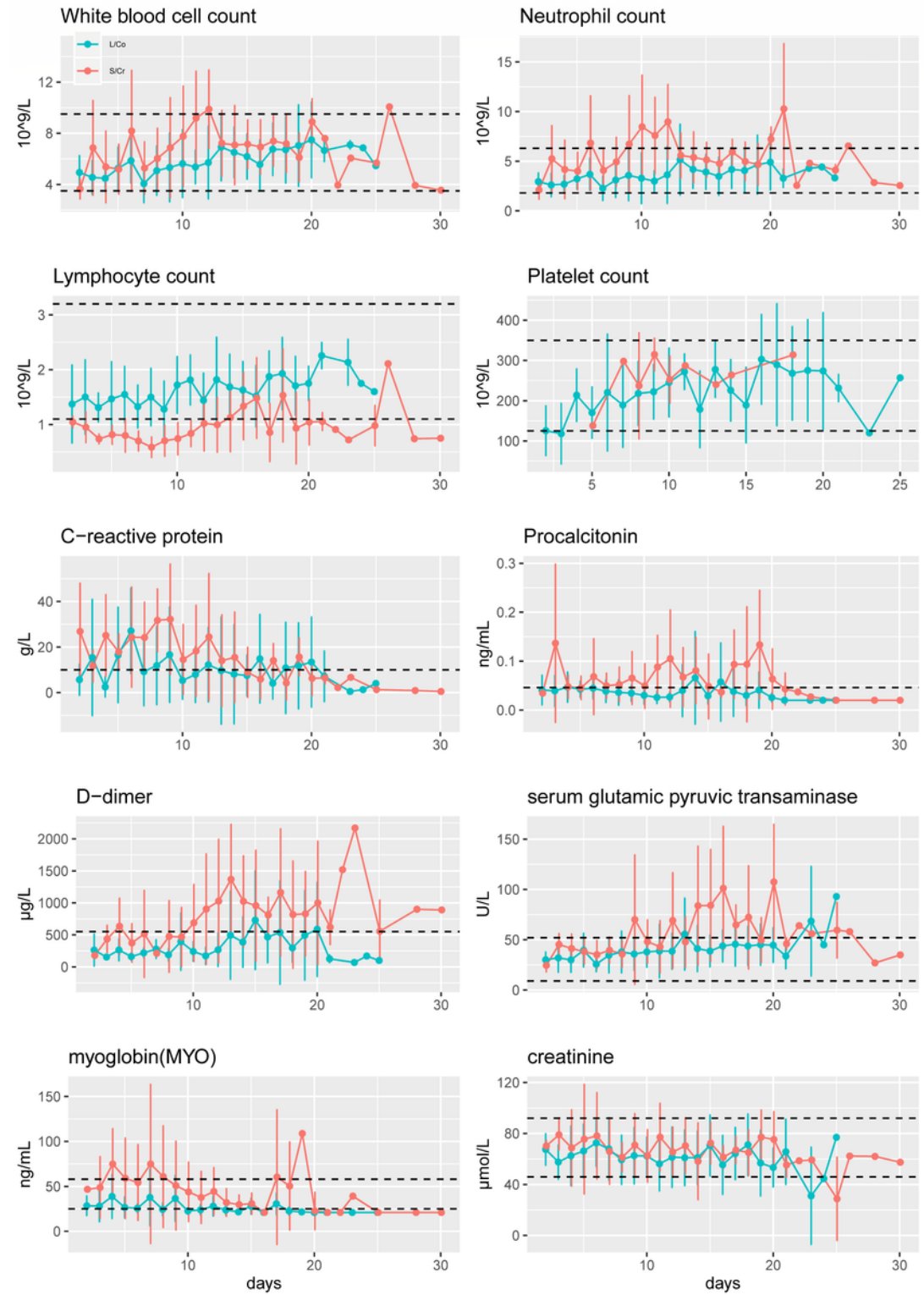

\section{Figure 1}

Dynamic profile laboratory parameters in 85 patients with COVID-19 without extreme value 

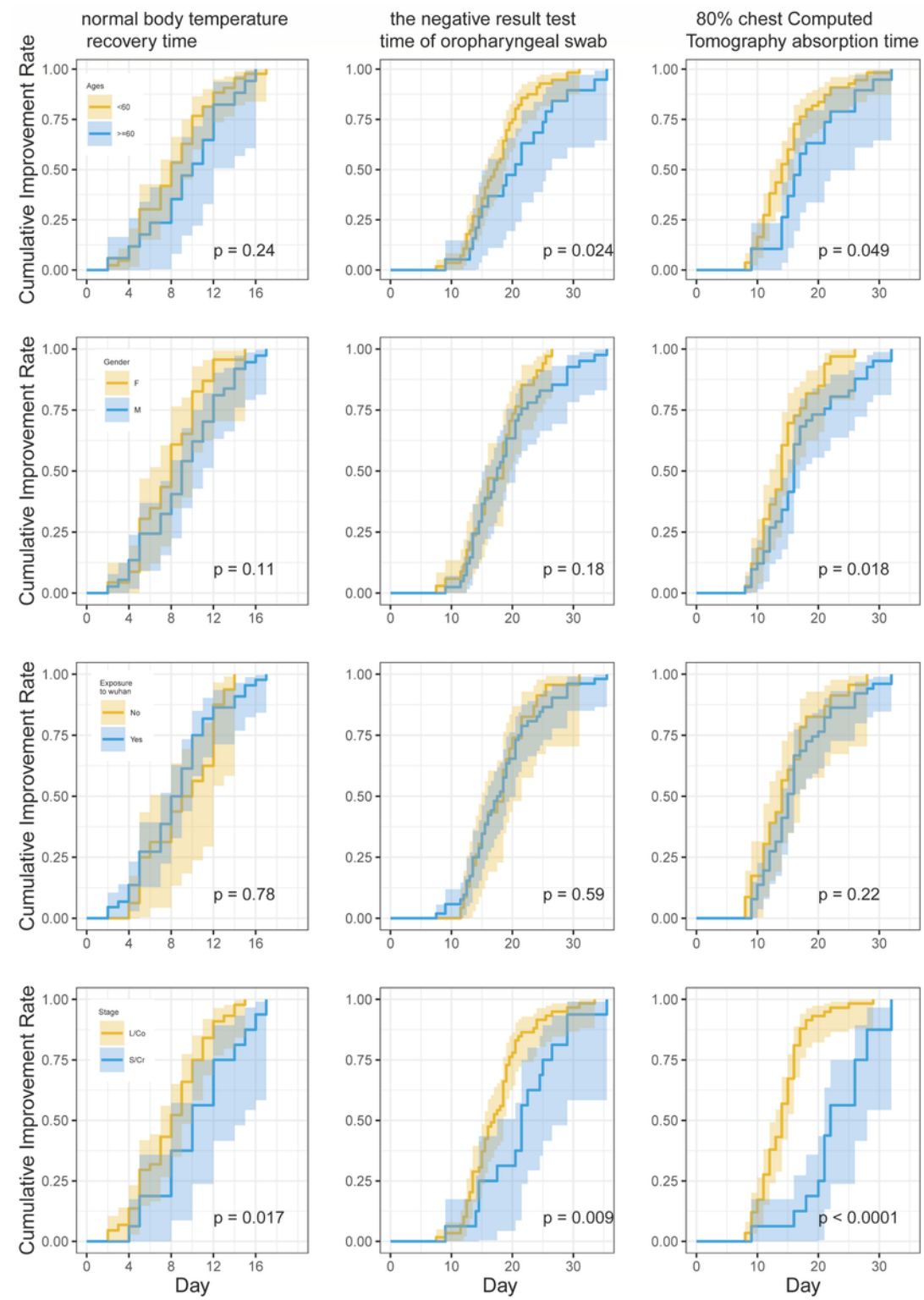

\section{Figure 2}

The correlation between clinical factor at baseline and clinical outcome event according to cumulative rate analysis 

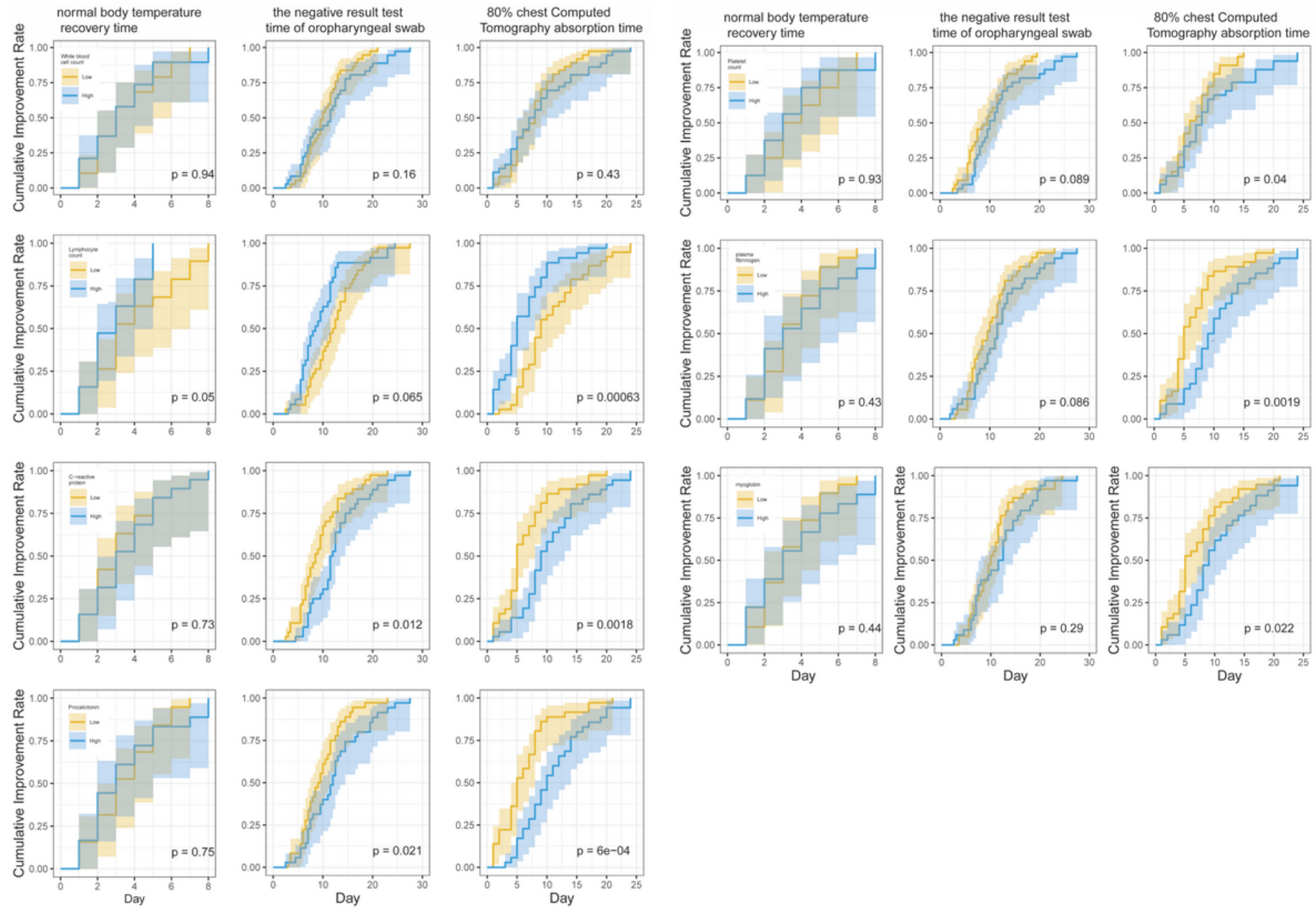

Figure 3

a. The correlation between clinical factor on the 8th onset and clinical outcome event according to cumulative rate analysis. b. The correlation between clinical factor on the 8th onset and clinical outcome event according to cumulative rate analysis

\section{Supplementary Files}

This is a list of supplementary files associated with this preprint. Click to download.

- SupplementaryFigure2ForestmapforcorrelationanalysisbetweenclinicalbaselinefactorandclinicaloutcomeeventsofCOVID19patients.tiff

- SupplementaryFigure1Dynamicprofilelaboratoryparametersin85patientswithCOVID19withextremevalue.tif

- supplementalTable1.complicationsandtreatmentofCOVID19patients.xlsx 